\title{
Analysis of the Superiority of Flexible Construction
}

\author{
Chengshui YU ${ }^{\mathrm{a}, 1}$ \\ ${ }^{a}$ CRCC Development Group Co.,LTD, Beijing 100041, China
}

\begin{abstract}
With the continuous development of the times and the continuous progress of science and technology, intelligent robots, big data, artificial intelligence, and other technologies are gradually emerging in the construction industry. Building construction inevitably needs new blood to solve current problems under the tide of information age. Intelligent building has become a necessary way for building development. At present, in the field of intelligent construction, research and theory in various directions have developed to varying degrees. Through the analysis of existing theories, this paper sorts out the theoretical framework and core logic of intelligent construction, and looks forward to the development trend of intelligent construction in the future.
\end{abstract}

Keywords. Flexible construction, digital technology, artificial intelligence, intelligent building

\section{Introduction}

Construction has been a labor-intensive industry since its inception. From semi-natural dwellings in ancient times to houses and palaces built with wooden and stone structures, to skyscrapers built with steel structures, concrete and other new materials that accommodate tens of thousands of people now, the building is still a labor-intensive project born from earth, wood, and brick. Architecture today not only provides the basic function of sheltering from wind and rain, but also meets the needs of people ' $\mathrm{s}$ modern life, large volume and diverse functional requirements make its construction a complex project requiring multidisciplinary collaboration. Building construction belongs to a special discrete manufacturing industry. Its production process consists of a series of discontinuous activities. Because construction products are customizable, they cannot form standard production organization plans like manufacturing [1]. During implementation, after the detailed construction plan in the process of execution, construction personnel, site conditions, materials, equipment, etc. are all in the changing environment, a variety of factors affecting the interaction between led to the complexity of the project and increase the degree of uncertainty, which caused the construction delay or resource conflicts, even cause the project is out of control [2]. In order to cope with the uncertainty of the construction process, there is often enough redundancy in the planning process, but this can lead to a waste of resources [3].

In addition, the architecture has more new forms different from the traditional, under the background of parameterization and nonlinear architecture forming a new

${ }^{1}$ Chengshui Yu, CRCC Development Group Co., LTD, Beijing 100041, China; E-mail: yuchengshui000@163.com. 
architectural style, and the new aesthetics became more and more accepted. The complex dynamic curves and flowing nonlinear shapes of these buildings also bring a lot of trouble to the construction. This puts forward higher requirements for construction and construction management. This puts forward higher requirements for construction and construction management.

With the arrival of the fourth industrial revolution, information technology industry has gradually penetrated every corner of the construction industry. Building information model, internet of things, big data, intelligent robot, cloud computing and other technologies have been gradually applied to the construction industry. Intelligent building looks like the most likely solution for the construction industry. At present, China's construction industry is developing continuously and rapidly, and its scale is getting bigger and bigger. But the management mode of building construction is not closely combined with advanced manufacturing technology and information technology, communication between the professions is not smooth, response to the chain reaction triggered by changes in the design phase is always slow, a lot of manpower and material resources were wasted, and construction safety problems cannot be solved well. At present, China has paid more and more attention to the development of intelligent construction and information technology, and flexible construction has responded to the problem of construction mode in intelligent construction.

\section{Concept of Flexible Construction}

Through the comprehensive application of various technologies in the intelligent era, flexible construction accurately controls all aspects of construction and production, and makes full use of digital technology to realize flexible construction mode in many aspects such as design, construction, management, docking and operation. Before the actual construction, flexible construction in advance through the digital technology, such as the generation of the digital model, construction of the wind, light, heat, and other physical environment simulation analysis, to provide design, analysis, coordination, construction, maintenance, and other all-round simulation, in order to found the problem in advance, minimize problems in the process of actual construction.

\section{Content of Flexible Construction}

Flexible construction aims to use information technology means, combined with fine construction and modular construction, to respond to the dynamic design process and constantly changing construction environment, to achieve the purpose of high efficiency, easy adjustment, high quality, resource saving, safe construction; through the overall system, partition modularization, solve the core technical difficulties, establish an effective construction organization, to achieve the overall arrangement of the design, materials, construction, funds, management of the construction project. The core of traditional construction is the civil engineering component, which requires much effort from the organization of construction to the integration of funds, materials, and manpower. Complex projects make the construction process inefficient. The core of flexible construction is communication, data, cloud computing, artificial intelligence, etc. It uses information technology to count the relevant data of each link, processes 
and analyses the data with the help of computer technology, and reserves space for the development and transformation of future modules, so that the building can meet the use needs of future functions.

Flexibility is generally understood as sensitivity to adaptation to rapidly changing environments. Flexibility in flexible construction refers to a flexible response to productive capacity and an agile response to instability in a changing construction market. Flexibility is a potential ability since construction costs, construction speed, construction quality, post-maintenance and other aspects of production are different, and no one model can perfectly adapt to all construction links, the flexible construction industry is also not a concrete system. The higher the system flexibility is, the wider the system can reach, the shorter the response time is, and the less the resources are consumed. The reduction of construction time and the cost of the construction from one construction state to another construction state all play an important role in the construction process. This also reflects the adaptability of construction patterns to state changes. The faster and smoother the transition from one system or state to another, the greater the benefits, the less wasted resources, and the more flexible the construction system is.

\section{Flexible Construction Methods}

Flexible construction includes lean construction, concurrent engineering, agile construction, and intelligent construction. Lean construction emphasizes the importance of effectively organizing the construction process to improve production efficiency and reduce waste of resources. Its core idea is to reasonably integrate existing construction resources from the perspective of overall optimization, and try to eliminate the influence of uncertain factors on the construction process [4]. Lean construction is based on a smooth flow of information within the organization, which requires real-time analysis of field information to make decisions; concurrent engineering refers to a multi-functional collaborative team that tracks the project and considers construction, maintenance, and management issues from the beginning of the design process; agile construction focuses on rapid response to changes in the external environment and responds to potential problems with dynamic organizational forms and complete technical support; intelligent construction covers the whole process from design to construction to management. The main technologies of intelligent construction include intelligent robots, $3 \mathrm{~d}$ printing and internet of things, which are of great help to improve the flexibility of construction mode.

Enterprises need more information technology applications for flexible construction, such as big data acquisition and processing, computer technology, cloud computing, etc. For enterprise, implements the flexible construction should have some conditions, such as transforming the traditional department system into an integrated team with architecture as the main line and a variety of professional assistance, with cross-disciplinary cooperation, small-scale and multi-skilled teams as the core mode to solve the problem of slow information flow caused by poor communication between different departments; establishing collaborative work environment to solve the problem that digital information cannot be exchanged directly between different links, developing multiple interfaces for the platform, the comprehensive use of modern management technology, information technology, the work of related personnel integrated into the same platform, so that the design, construction, management 
information can be barrier-free circulation, update quickly; set up enterprise alliance to make up for the problem of insufficient resources of a single enterprise, and to improve the overall flexible construction ability through collaborative work and resource sharing supported by computer.

Construction personnel also need to master more information technology. Due to the changes in the construction mode, more and more types of work will be undertaken by the construction personnel; for the project management personnel, it is necessary to have a certain grasp of the dynamic analysis ability of construction and the automated office ability.

\section{Key Technologies of Flexible Construction}

Information processing and visualization. Information processing occupies a very important position in flexible construction. A large number of data are analyzed by means of real-time communication, big data and cloud computing, and the analysis results are used for decision-making. In this process, information visualization can serve as a bridge between virtual data and reality. For example, using information visualization technology such as virtual reality and augmented reality technology, accurate design, and construction simulation of the project in the digital environment, predict the future construction process, find possible problems, avoid, and prepare for the corresponding risk in advance [5].

Artificial intelligence. The role of artificial intelligence in flexible construction is to assist decision-making. Through the use of machine learning, artificial neural network, probability calculation and other technologies and algorithms, and the analysis and learning of a large number of cases and data, the system can replace manual work to complete many tasks such as preliminary analysis, intelligent planning, intelligent design, and risk prediction.; in the flexible construction system, artificial intelligence can analyze the collected data, update and adjust the changes in time, optimize the construction process and assist decision-making, so as to enhance the flexibility of the system (as shown in figure 1).

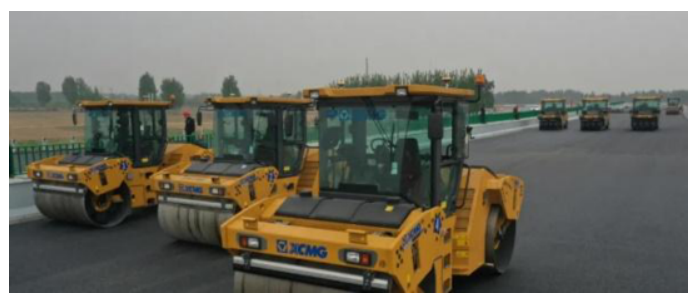

Figure 1. Automated paving construction.

Intelligent robot. Flexible construction meets diverse construction requirements, and intelligent robots have great potential in this regard. Robots controlled by computer programming can process and construct complex and nonlinear shapes. According to different control procedures, intelligent robots can simultaneously construct various architectural components with different shapes and complete diversified work. This saves more time and effort than traditional construction and improves construction accuracy and quality (as shown in figure 2) [6]. 


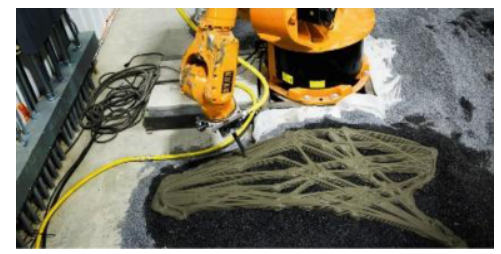

Figure 2. 3d print of curved concrete lattice structures by Cornell University.

Collaborative work platform. Through the construction of digital work platform to solve the problem of communication between different links. Through the development of the platform port to make it compatible with a variety of digital information and models, and establish a collaborative work environment to improve the system response speed and improve system flexibility.

\section{Advantages of Flexible Construction}

The advantages of flexible construction:

Meet a variety of architectural needs. Digital technology changes architects' design thinking. Building shapes generated by various algorithms are different from traditional regular modelling, such as optimal structure under topology analysis, minimal surface of continuous flow, streamline generation under shortest path, etc. With the prevalence of parameterism and nonlinear architecture, architecture is becoming more and more personalized and customized. A variety of large span, large cantilever and complex buildings require non-standardized building components. The traditional construction mode has limited construction capacity for these buildings, and the small batch production of components will consume more resources, while the construction components produced in batches sometimes cannot meet the needs of architectural development. Flexible construction can produce different building products with the change of products, and it does not need to consume more resources in a small output to meet the diversified construction needs.

Flexible process. Usually, the same production line can only produce one component in traditional construction, if there are some changes in the component, such as minor changes only somewhere in the component, the whole process of the production line needs to be changed. In the case of small but many types of changes, the traditional construction model is not capable, needs a lot of time, and cannot achieve its expected economic benefits. Flexible construction can change the original construction process according to the change of the machining object, and switch to another production mode in time. It can quickly adjust the components with multiple changes and types to produce the required components. Rapid response capability greatly improves construction efficiency, shortens construction period, and reduces waste of resources.

Flexible production. Information technology, such as cloud computing, visual management, artificial intelligence, etc., is utilized to collect, analyze, and predict the construction process and market data in real time, and feedback the information to the links of construction, material preparation, construction, and production. When production changes, the system can respond in a timely manner by adjusting its production capacity to adapt to new production needs and feeding back the adjusted 
capacity to the system in a timely manner to keep the construction process smooth and the production materials economical.

Flexibility of expansion capability. When production needs, the existing production structure can be easily extended to increase production by adding modules to form a larger production system.

Flexibility of management. The system can adjust the project clearly and intuitively in many ways, and quickly respond to changes in the design and construction process. Through the analysis of market data, the production mode can be adjusted in time to ensure that the design source matches the design purpose and market, so that the project is always in a normal, efficient, and economic operation mode.

\section{Conclusions}

Construction industry is an ancient industry, but also the pillar industry of the national economy, in the information age, architecture also needs to be combined with the development of the times, respond to the trend of the development of the times, and actively change. Under the development trend of intelligent construction, flexible construction has its unique advantages. It is a targeted change to the problems existing in the traditional construction, and a more reasonable use of social resources. It is worthy of further research.

\section{References}

[1] You Z, Zheng L, Feng L. Basic theory and architecture of intelligent construction system. Journal of Civil Engineering and Management. 2021 Apr; 38(2):105-111.

[2] Ding L, Qi S, Chen F. Design and realization of large-complex construction engineering intelligent schedule plan management system. Construction Technology. 2006 Dec; 35(12): 121-123.

[3] Oesterreich T, Teuteberg F. Understanding the implications of digitisation and automation in the context of industry 4.0: A triangulation approach and elements of a research agenda for the construction industry. Computers in Industry. 2016 Dec; 83: 121-139

[4] Ansah H, Sorooshian S, Mustafa. Lean construction: An effective approach for project management. ARPN Journal of Engineering and Applied Sciences. 2016 Jan; 11(3):1607-12

[5] Luo D, Xu W. Virtual reality in architectural practice. Architecture Technique. 2017 Sept; 9: 75-77.

[6] Yuan F, Menges A. Building robotics: technology, craft and methodology. In: Zhao X, Zhu X, Zhang Z 1st, editors. Introduction to building Robots. Beijing: China Architecture \& Building Press; 2009. p. 811. 